\title{
The molecular classification of astrocytic tumors
}

\author{
Chen-Xue Mao $^{1,3}$, Ji-Ye Yin ${ }^{1,3}$, Ying Zhang, ${ }^{1,3}$, Zhi-Bin Wang ${ }^{1,3}$, Zhi-Quan Yang ${ }^{2}$, \\ Zheng-Wen $\mathrm{He}^{4}$, Xiang-Min Li ${ }^{5}$, Xiao-Yuan Mao ${ }^{1,3}$, Ru-Tao Cui ${ }^{6}$, Xue-Jun Li ${ }^{2}$, Xi Li ${ }^{1,3}$, \\ Wei Zhang ${ }^{1,3}$, Hong-Hao Zhou ${ }^{1,3}$ and Zhao-Qian Liu ${ }^{1,3}$ \\ ${ }^{1}$ Department of Clinical Pharmacology, Xiangya Hospital, Central South University, Changsha 410008, P. R. China \\ ${ }^{2}$ Department Neurosurgery, Xiangya Hospital, Central South University, Changsha 410008, P. R. China \\ ${ }^{3}$ Institute of Clinical Pharmacology, Central South University, Hunan Key Laboratory of Pharmacogenetics, Changsha 410078, \\ P. R. China \\ ${ }^{4}$ Department of Neurosurgery, The Affiliated Cancer Hospital of Xiangya School of Medicine, Central South University, \\ Changsha 410014, P. R. China \\ ${ }^{5}$ Department of Emergency, Xiangya Hospital, Central South University, Changsha 410008, P. R. China \\ ${ }^{6}$ Departments of Pharmacology and Experimental Therapeutics, Boston University School of Medicine, Boston, MA 02118, \\ USA \\ Correspondence to: Zhao-Qian Liu, email: liuzhaoqian63@126.com \\ Keywords: astrocytoma, calcium signaling pathway, IDH, molecular classification, chemokine signaling pathway \\ Received: July 29, $2017 \quad$ Accepted: August 23, $2017 \quad$ Published: October 25, 2017 \\ Copyright: Mao et al. This is an open-access article distributed under the terms of the Creative Commons Attribution License 3.0 \\ (CC BY 3.0), which permits unrestricted use, distribution, and reproduction in any medium, provided the original author and source \\ are credited.
}

\section{ABSTRACT}

Aim: This study will explore the genetic and epigenetic alterations in astrocytomas, and identify the critical molecular signatures and signaling pathways for prognosis assessment by multiplatform comprehensive analysis.

Method: We performed integration analyses of incorporating DNA methylation, mRNA expression, microRNA expression, and long non-coding RNA (IncRNA) expression in $\mathbf{3 3}$ astrocytic tumor tissues and 9 non-tumor brain tissues.

Result: We observed that 11,795 DNA methylation sites, 3,627 genes, 136 microRNAs, and 3,334 IncRNAs were significantly differential between tumors and non-tumor brain tissues, and the filtered signatures through comprehensive analysis were significantly enriched in calcium signaling pathway. Furthermore, four signatures involved in calcium signaling pathway and age could contribute to predicting the patients' overall survival. Additionally, we identified differentially expressed signatures between IDH-mutated and IDH wild-type astrocytic tumors, and complement and coagulation cascades pathway was the most significant pathway in functional enrichment analysis using multiplatform data. The IDH wild-type astrocytomas were divided into two subtypes by Cluster of Cluster (CoC) analysis, one of which was enriched for astrocytomas overexpressed in chemokine signaling pathway.

Conclusion: The calcium signaling pathway played a key role in astrocytoma tumorigenesis and prognosis. IDH mutation was a vital biomarker, and resulted in the change of expression level in complement and coagulation cascades pathway. The chemokine signaling pathway could characterize subtypes of IDH wild-type astrocytomas. 


\section{INTRODUCTION}

Among primary central nervous system tumors, gliomas are the largest and most diverse group [1]. Gliomas can be classified as astrocytomas, oligodendrogliomas or oligoastrocytomas and graded as I to IV according to the histopathological classification of World Health Organization [2]. Glioblastoma (GBM), the grade IV astrocytoma, arises most frequently and has the worst prognosis with median survival of 1 year [3]. The widely infiltrative properties make astrocytoma impossible to be completely resected and likely to malignantly progress and recur, resulting in highly variable survival periods of patients. The single traditional diagnostic method based on histopathologic characteristics cannot satisfy the requirement of predicting clinical outcomes [4-6].

More recently, understanding of genetic biomarker has been improved along with the discovery of numerous molecular alterations in gliomas. Some molecular alterations are now recognized as major biomarkers of clinical behavior and molecular classification, including IDH mutation, $1 \mathrm{p} / 19 \mathrm{q}$ codeletion, TERT mutation and MGMT methylation [7-13]. However, the genetic and epigenetic characteristics of astrocytomas have not been extensively explored by multiplatform comprehensive analysis.

In the present study, we investigated the DNA methylation and the expression level of genes, miRNAs, and long non-coding RNAs (lncRNAs) in 33 astrocytic tumor tissues and 9 non-tumor brain tissues by microarray analysis, predicted the potential biological function of significantly differentially expressed signatures by integration analysis of multiplatform data, and assessed the prognostic value. We also observed the effect of IDH mutation on DNA methylation and gene expression level. Finally, we constructed a molecular classification of IDH wild-type astrocytomas and identified the biomarker of classification.

\section{RESULTS}

\section{Clinical characteristics of the astrocytoma cases}

We found IDH mutations in $30.30 \%$ of astrocytomas samples and $51.51 \%$ for TERT mutations, with one sample having both mutations. Differences in the prevalence of IDH mutation and MGMT promoter methylation between lower-grade astrocytomas and grade IV GBM were significant. Nearly all astrocytomas with IDH mutation were lower-grade astrocytomas, and the prevalence of MGMT promoter methylation in lower-grade astrocytomas was higher than that in GBM ( $\mathrm{p}=0.032$, Fisher's exact test). Samples of astrocytomas with MGMT promoter methylation had less TERT mutations than samples with low MGMT promoter methylation $(\mathrm{p}=0.041)$. Previous studies showed that $1 \mathrm{p} / 19 \mathrm{q}$ codeletion occurred most often in oligodendroglial gliomas. We identified only 5 astrocytomas with $1 \mathrm{p} / 19 \mathrm{q}$ codeletion in this study, and they all were IDH wild-type and MGMT promoter methylation samples. The clinical information of patients is provided in Table 1 .

\section{Identification of differential signatures between astrocytic tumors and non-tumor brain tissues}

First, a total of 11,795 probes were identified as differentially methylated by the criteria of corrected p-value $<0.05$ and absolute $\beta$-difference $>0.2$ between 33 astrocytic tumors and 9 non-tumor brain tissues (Supplementary Figure 1B). Then, the two-dimensional hierarchical clustering was performed using the top 5000 differentially methylated probes between tumors and NTL tissues (Supplementary Figure 2B). Finally, we tested 6 DNA methylation probes using pyrosequencing in 65 astrocytic tumors and 16 non-tumor brain tissues. The results demonstrated that 4 probes were significantly hypermethylated and 2 probes were significantly hypomethylated in astrocytic tumors (Supplementary Figure 3), which is consistent with microarray results.

Moreover, we identified 3,627 genes, 3,334 lncRNAs and 136 miRNAs differentially expressed by the criteria of corrected p-value $<0.05$ and fold change $\geq 2$ in 33 astrocytic tumors and 9 non-tumor brain tissues (Supplementary Figure 1A, 1C and 1D). The two-dimensional hierarchical clustering was performed using the differentially expressed genes, lncRNAs and miRNAs, respectively (Supplementary Figure 2A, 2C and 2D). All heatmaps revealed clear separation of tumors and NTL tissues. The expression of 10 genes, 2 miRNAs and 6 lncRNAs in astrocytic tumors and non-tumor brain tissues were validated by quantitative real-time PCR (Supplementary Figures 4-6), and the results coincided with that of microarrays.

By integrating the gene expression profiles and DNA methylation profiles, 552 genes were statistically significantly hypermethylated and down-regulated, while 297 genes were significantly hypomethylated and upregulated (Figure 1A). We identified 196 hypermethylated and down-regulated genes showing strongly inverse correlation between DNA methylation and gene expression by calculating the Pearson correlation coefficients (PCC) $(\mathrm{PCC}<-0.5)$. Among these genes, ANK3 was ranked highest based on the PCC of three methylation sites ranging from -0.75 to -0.87 (cg22150335, cg20135031 and cg14391247) (Supplementary Figure 7A-7C). In addition, TSPYL5, RAB3A, ABCA2, NCDN and DLGAP3 were also noteworthy. Scatterplots of these genes expression versus DNA methylation are shown in (Supplementary Figure 7D-7L).

Potential gene targets for each dysregulated miRNA were obtained by miRWalk (http://www.umm. 
Table 1: Clinical characteristics of patients

\begin{tabular}{|c|c|c|c|}
\hline Characteristic & Classes & Tumor & Non-tumor \\
\hline \multirow{2}{*}{ Age } & $\leq 50$ & $22(66.67 \%)$ & $3(33.33 \%)$ \\
\hline & $>50$ & $11(33.33 \%)$ & $6(66.67 \%)$ \\
\hline \multirow{2}{*}{ Gender } & Male & $20(60.61 \%)$ & $7(77.78 \%)$ \\
\hline & Female & $13(39.39 \%)$ & $2(22.22 \%)$ \\
\hline \multirow{2}{*}{ IDH mutation } & wild type & $23(69.70 \%)$ & \\
\hline & mutation & $10(30.30 \%)$ & \\
\hline \multirow{3}{*}{ TERT promoter mutation } & wild type & $14(42.42 \%)$ & \\
\hline & mutation & $17(51.51 \%)$ & \\
\hline & absent & $2(6.06 \%)$ & \\
\hline \multirow{2}{*}{$1 \mathrm{p} / 19 \mathrm{q}$ codeletion } & no codeletion & $28(84.85 \%)$ & \\
\hline & codeletion & $5(15.15 \%)$ & \\
\hline \multirow{2}{*}{ Grade } & II-III & $17(51.52 \%)$ & \\
\hline & IV & $16(48.48 \%)$ & \\
\hline \multirow{2}{*}{ MGMT methylation } & Low & $12(36.36 \%)$ & \\
\hline & High & $21(63.64 \%)$ & \\
\hline
\end{tabular}

uniheidelberg.de/apps/zmf/mirwalk), which combined prediction results of different algorithms including DIANAmT, miRanda, miRDB, miRWalk, RNAhybrid, PICTAR4, PICTAR5, PITA, RNA22 and Targetscan. The predicted gene targets were specifically identified by at least 3 of these programs. Then, the Pearson correlation coefficients (PCC) between expression of the miRNA and their target genes were calculated. Finally, we identified 6,716 miRNA-gene co-expression pairs composed of 103 miRNAs and 1,645 genes with $\mathrm{PCC}<-0.7$ or $>0.7$ and $\mathrm{p}$-value $<0.05$. In addition, for each dysregulated lncRNA, the Pearson correlation coefficients (PCC) of its expression and that of each dysregulated gene was calculated. 70,948 lncRNA-gene co-expression pairs composed of 2,419 lncRNAs and 2,848 genes were found by $\mathrm{PCC}<-0.8$ or $>0.8$ and $\mathrm{p}$-value $<0.05$. Taken together, 315 genes overlapped in integration analyses were selected for further analysis.

The Gene Ontology (GO) and Kyoto Encyclopedia of Genes and Genomes (KEGG) pathway analysis was performed to determine the biological functions of these 315 genes by DAVID database (http://david.abcc.ncifcrf. gov/). The set of 315 genes was significantly enriched in KEGG pathways including calcium signaling pathway (Benjamini-Hochberg adjusted p-value $=0.029$ ) and neuroactive ligand-receptor interaction (BenjaminiHochberg adjusted $\mathrm{p}$-value $=0.042$ ) (Table 2). The findings suggested that calcium signaling pathway played a key role in astrocytoma, and the genes of calcium signaling pathway in the 315 gene set were listed in Supplementary Table 4. The hierarchical clustering analysis of the significantly differential signatures involved in calcium signaling pathway was shown in Figure 1A.

\section{Survival prediction analysis by the signatures of calcium signaling pathway}

To validate multiplatform differentially expressed signatures involved in calcium signaling pathway were significantly associated with overall survival of astrocytoma patients, we did univariate Cox regression analysis using multiplatform data of 92 GBM samples in TCGA.

We identified 13 DNA methylation probes, 2 genes, 2 miRNAs involved in calcium signaling pathway and age that were independently associated with overall survival. After adjusted multivariate Cox regression analysis for the age, 9 DNA methylation probes, 1 gene, 2 miRNAs were significantly correlated with overall survival. To establish a prediction model, multivariable Cox regression analysis was performed using a backward stepwise method, and we selected CHRM2 cg02866106, CACNA1B cg05863502, CALM1 cg18079499, miR-183 and age to construct the prognostic model. We established a formula comprised of the 4 signatures (correlated with good prognosis) and age (correlated with poor prognosis) to calculate the risk score for every patient. Risk score $=(0.055 \times$ age $)-(1.517 \times$ beta value of CHRM2 $\operatorname{cg} 02866106)-(1.398 \times$ beta value of CACNA1B cg05863502) - (5.060 × beta value of CALM1 cg18079499) - (0.584 $\times$ expression value of miR-183) By the cutoff of median risk score $(-3.058)$, patients were divided into high-risk or low-risk groups according to the 
Table 2: The top significantly enriched KEGG pathways in integration analyses of tumor vs. non-tumor tissues

\begin{tabular}{lcc}
\hline KEGG pathway & p-value & BH adjusted p-value \\
\hline Calcium signaling pathway & $2.388 \times 10^{-4}$ & 0.029 \\
Neuroactive ligand-receptor interaction & $7.003 \times 10^{-4}$ & 0.042 \\
Glioma & $9.285 \times 10^{-3}$ & 0.316 \\
Long-term potentiation & 0.013 & 0.322 \\
ErbB signaling pathway & 0.033 & 0.561 \\
Prion diseases & 0.035 & 0.512 \\
\hline
\end{tabular}

value of risk score. Kaplan-Meier analysis showed that patients in high-risk group had significantly shorter overall survival than patients in low-risk group (median survival 8 months vs 18 months, $\mathrm{p}<1 \times 10^{-4}$ ) (Figure 1D).
(A)

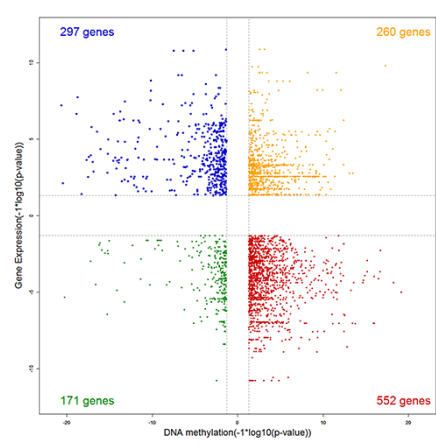

(B)

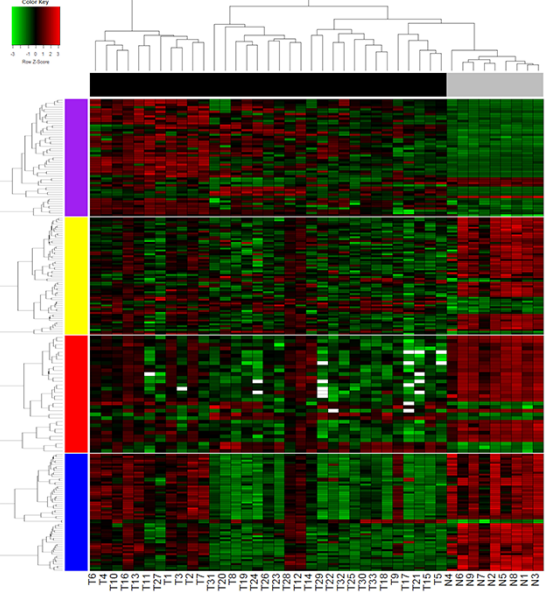

(D)
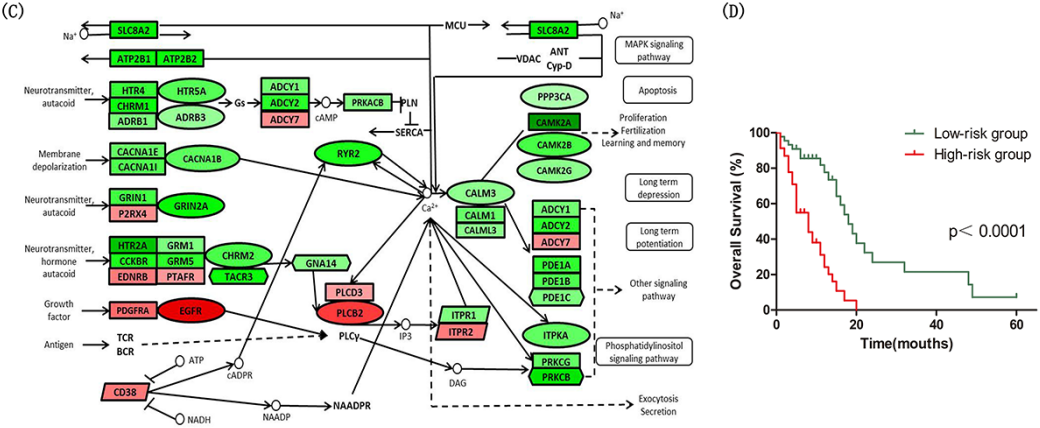

Figure 1: Identification of differential signatures between astrocytic tumors and non-tumor brain tissues. (A) Starburst plot integrating differential DNA methylation and gene expression analyses. Indicated are genes that are hypermethylated and downregulated in tumors (red); hypomethylated and up-regulated in tumors (blue); hypermethylated and up-regulated in tumors (yellow); or hypomethylated and down-regulated in tumors (green). (B) Two-dimensional hierarchical clustering of the significantly differential signatures in calcium signaling pathway in all samples (33 tumor tissues in black vs. 9 NTL tissues in gray). Signatures are in rows (51 differentially methylated and gene expression direction inversed probes in purple; 62 differentially expressed genes in yellow; 32 differentially expressed miRNAs in red; 59 differentially expressed lncRNAs in blue); samples are in columns. (C) Schematic representation of calcium signaling pathway. Ellipses represent genes overlapped in differential signatures of four platforms; rectangles represent genes within miRNA-gene co-expression pairs; parallelograms represent genes within IncRNA-gene co-expression pairs but not within miRNA-gene co-expression pairs; hexagons represent genes significantly hypermethylated and down-regulated or significantly hypomethylated and up-regulated, but not within miRNA-gene co-expression pairs. Gene color corresponds to fold change (genes colored in red are up-regulated, genes colored in green are down-regulated). (D) Kaplan-Meier curves of overall survival in astrocytoma patients. Patients were classified into high-/lowrisk group according to the risk score formula combining signatures involved in calcium signaling pathway and age. 


\section{Identification of differential signatures between IDH-mutated and IDH wild-type astrocytic tumors}

To identify subtypes and the vital biomarker of astrocytomas, we unsupervised clustered multiplatform data on 33 astrocytoma tumors.

The median absolute deviation (MAD) was calculated for each DNA methylation probe and the top 3,000 probes were selected for non-negative matrix factorization (NMF) clustering analysis. The NMF clustering and rank estimates were calculated for ranks 2-5 with default settings of method brunet and seed random. A two-cluster result was determined by rank survey profiles for cophenetic coefficient and visual inspection of the correlation matrices (Supplementary
Figure 8B). By the same method, the 3,000 genes, 150 miRNAs and 1,500 lncRNAs with the highest median absolute deviation (MAD) were defined as three clusters, respectively (Supplementary Figure 8A, 8C and 8D). In all unsupervised clustering analysis results, one of the clusters was closely related to IDH mutation. The consistent results indicated that IDH mutation could define a subtype of astrocytoma.

To determine the effect of IDH mutation on DNA methylation and RNA expression, we did a differential analysis between 10 IDH-mutated and 23 IDH wildtype astrocytic tumors, and 28,609 DNA methylation probes, 1,862 genes, 42 miRNAs and 1,734 lncRNAs were identified differentially expressed in total. These differentially expressed signatures were used to perform hierarchical clustering analysis respectively, and all the
(A)

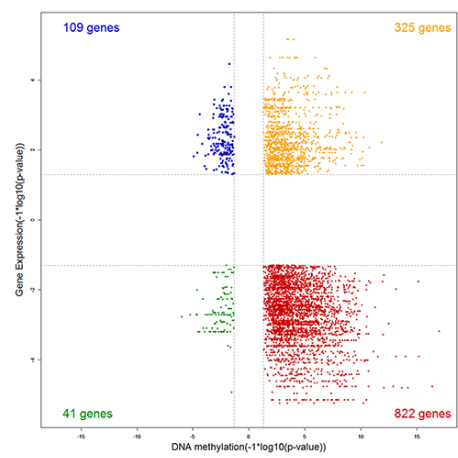

(C)
(B)

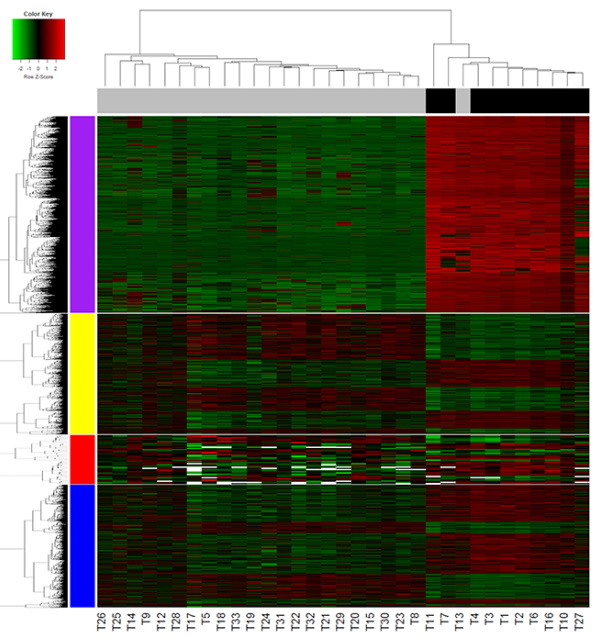

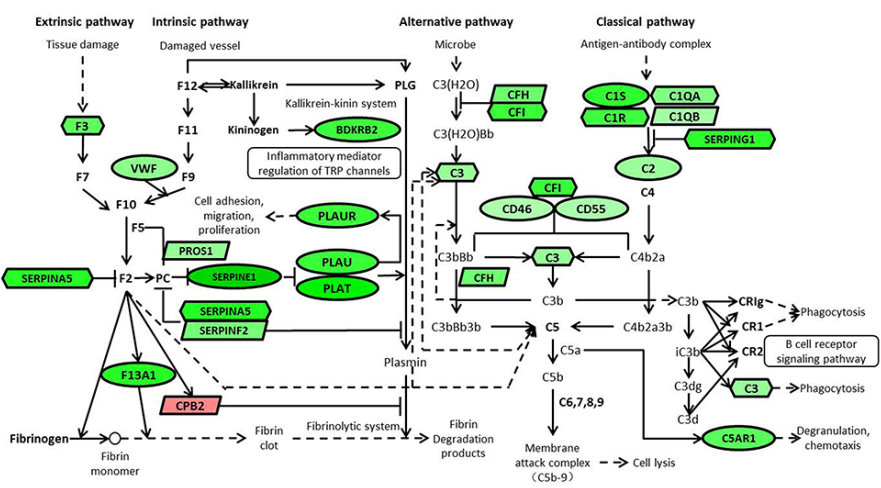

Figure 2: Identification of differential signatures between IDH-mutated and IDH wild-type astrocytic tumors. (A) Starburst plot integrating differential DNA methylation and gene expression analyses. Indicated are genes that are hypermethylated and down-regulated in IDH-mutated tumors (red); hypomethylated and up-regulated in IDH-mutated tumors (blue); hypermethylated and upregulated in IDH-mutated tumors (yellow); or hypomethylated and down-regulated in IDH-mutated tumors (green). (B) Two-dimensional hierarchical clustering of the significantly differential signatures in IDH-mutated astrocytic tumors (10 IDH-mutated astrocytic tissues in black vs. $23 \mathrm{IDH}$ wild-type astrocytic tissues in gray). Signatures are in rows (top 5,000 differentially methylated probes in purple; 1,862 differentially expressed genes in yellow; 42 differentially expressed miRNAs in red; 1,734 differentially expressed lncRNAs in blue); samples are in columns. (C) Schematic representation of complement and coagulation cascades pathway. Ellipses represent genes overlapped in differential signatures of four platforms; rectangles represent genes within miRNA-gene co-expression pairs; parallelograms represent genes within IncRNA-gene co-expression pairs but not within miRNA-gene co-expression pairs; hexagons represent genes significantly hypermethylated and down-regulated or significantly hypomethylated and up-regulated, but not within miRNA-gene coexpression pairs. Gene color corresponds to fold change (genes colored in red are up-regulated, genes colored in green are down-regulated). 
Table 3: The top significantly enriched KEGG pathways in integration analyses of IDH-mutated vs. IDH wild-type tumors

\begin{tabular}{lcc}
\hline KEGG pathway & p-value & BH adjusted p-value \\
\hline Complement and coagulation cascades & $2.259 \times 10^{-8}$ & $2.417 \times 10^{-6}$ \\
ECM-receptor interaction & $1.716 \times 10^{-6}$ & $9.179 \times 10^{-5}$ \\
Focal adhesion & $9.186 \times 10^{-6}$ & $3.276 \times 10^{-4}$ \\
Cell adhesion molecules (CAMs) & 0.029 & 0.542 \\
p53 signaling pathway & 0.032 & 0.498 \\
Viral myocarditis & 0.036 & 0.483 \\
\hline
\end{tabular}

heatmaps showed good separation of IDH-mutated and IDH wild-type astrocytic tumors in general (Figure 2B).

By the integration analysis of multiplatform data as described above, we found the signatures involving complement and coagulation cascades pathway significantly changed in IDH-mutated astrocytomas (Benjamini-Hochberg adjusted p-value $=2.417 \times 10^{-6}$ ) (Table 3). The differentially expressed genes between 6 IDH-mutated and 83 IDH wild-type GBM were also significantly associated with complement and coagulation cascades pathway in TCGA dataset (Benjamini-Hochberg adjusted $\mathrm{p}$-value $=0.010)($ Supplementary Table 5$)$.

\section{The molecular classification of IDH wild-type astrocytomas}

To construct molecular classification in IDH wild-type astrocytomas, we calculated the MAD value in multiplatform data and selected the top 3,000 DNA methylation probes, 1,500 genes, 150 miRNAs and 3,000 lncRNAs for NMF analysis, respectively. Unsupervised clustering identified two stable clusters in DNA methylation (M1, M2) (Supplementary Figure 9B), mRNA $(\mathrm{m} 1, \mathrm{~m} 2)$ (Supplementary Figure 9A), miRNA (mi1, mi2) (Supplementary Figure 9C) and lncRNA (lnc1, lnc2) (Supplementary Figure 9D) expression data from $23 \mathrm{IDH}$ wild-type astrocytoma samples. TERT promoter mutation was predominantly a feature of M1 DNA methylation cluster, $88.89 \%$ of samples in M1 cluster harbored a TERT promoter mutation. M1 cluster also was highly enriched for histologic grade IV GBM. 1p/19q codeletion was found in 5 samples, 4 of which were classified in mi2 cluster.

Clusters defined from each platform (DNA methylation, mRNA, miRNA, and lncRNA) were formed into a matrix of $1 \mathrm{~s}$ and $0 \mathrm{~s}$. The multiplatform data matrix was unsupervised consensus clustered using the Consensus ClusterPlus R-package (80\% sample resampling, 1000 iterations, and Pearson correlation). The robust number of $\mathrm{CoC}$ clusters was determined to be 2 using $\mathrm{K}$-means clustering algorithm for clusters ranging 2-7. Based on this, we divided IDH wild-type astrocytomas into two clusters using multiplatform data (Figure 3A).

The significance analysis of microarrays (SAM) method was applied to identify differentially expressed genes of each cluster, and the threshold of statistical significance was q-value $<0.1$. The highly expressed genes in $\mathrm{CoC} 1$ Cluster were significantly enriched in chemokine signaling pathway (Benjamini-Hochberg adjusted $\mathrm{p}$-value $=0.012$ ).

Then, we divided 83 GBMs with wild-type IDH in TCGA dataset into two groups by $\mathrm{CoC}$ analysis, and found that differentially expressed genes between two clusters were also significantly associated with chemokine signaling pathway (Benjamini-Hochberg adjusted p-value $=0.004)$ (Figure 3B).

To assess the prognostic value of signatures in chemokine signaling pathway, we did univariate Cox regression analysis using DNA methylation, mRNA expression, miRNA expression and survival data of 83 GBM with wild-type IDH in TCGA dataset. The DNA methylation levels of PRKCB cg03217795 and CXCL2 $\operatorname{cg} 23244559$, and the expression level of GNG13 were found significantly associated with overall survival. We derived a formula for these signatures and age with the same risk-score method as described above. The risk score of overall survival $=(0.054 \times$ age $)-(2.155 \times$ beta value of PRKCB cg03217795) - (1.974 $\times$ beta value of CXCL2 $\operatorname{cg} 23244559)+(0.359 \times$ expression value of GNG13). The astrocytoma patients with wild-type IDH were respectively classified as low- or high-risk groups of overall survival. Kaplan-Meier analysis showed that patients in low-risk group of overall survival survived significantly longer than patients in high-risk group (median survival 17 months vs 6 months, $\mathrm{p}<1 \times 10^{-4}$ ) (Figure $\left.3 \mathrm{C}\right)$.

\section{DISCUSSION}

Using an integrative, multiplatform approach, we found that calcium signaling pathway played a key role in astrocytoma tumorigenesis, and IDH mutation resulted in the change of gene expression in complement and 
coagulation cascades pathway. We also determined that chemokine signaling pathway could be the biomarker of molecular classification in IDH wild-type astrocytomas to predict the overall survival.

We identified 11,795 DNA methylation sites, 3,627 genes, 3,334 lncRNAs and 136 miRNAs showing significantly differential in astrocytic tumors. In addition, we found some hypermethylated genes with an associated decrease in expression, such as ANK3 [14, 15], TSPYL5 $[16,17]$, RAB3A and ABCA2 [18] that have been reported to be related with cancer or central nervous system diseases. Through a comprehensive analysis, DNA methylation, genes, miRNAs and lncRNAs involved in calcium signaling pathway were shown obviously altered

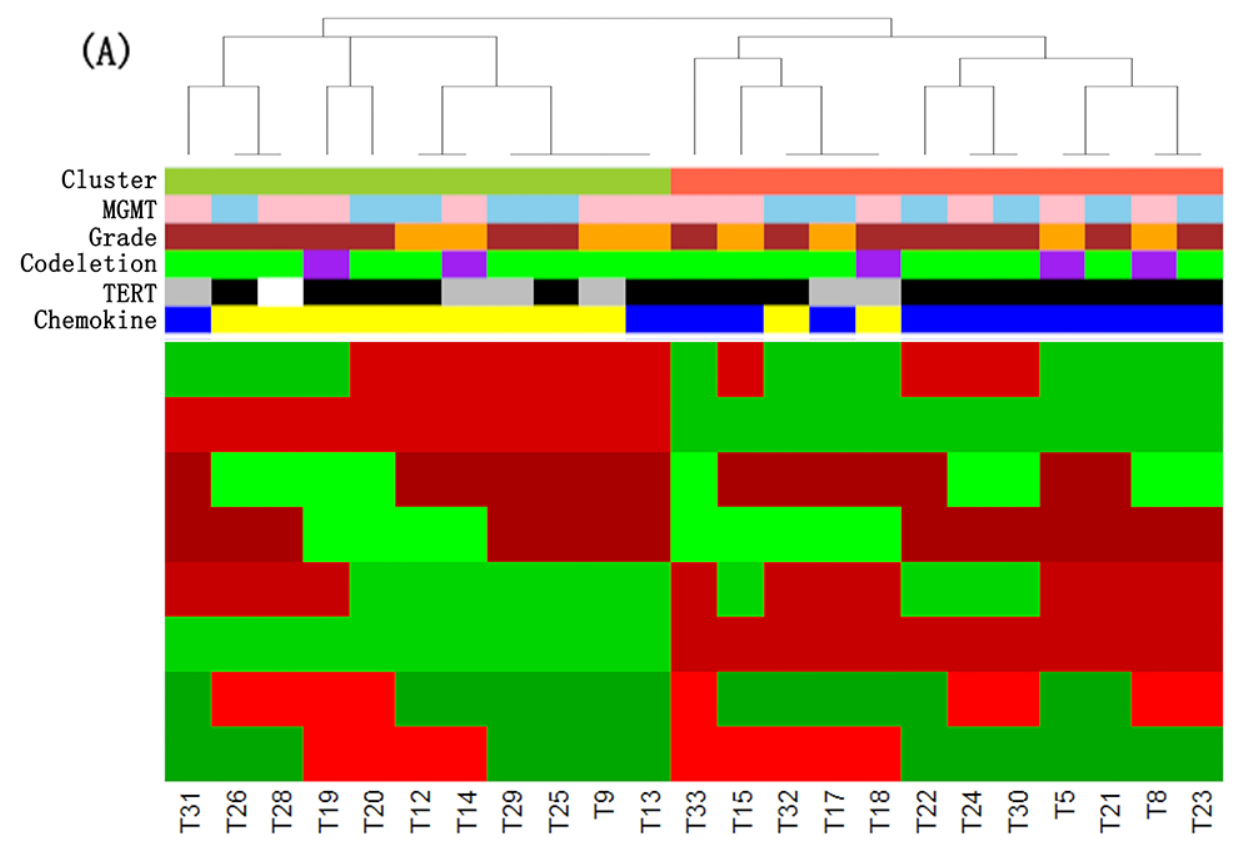

(B)

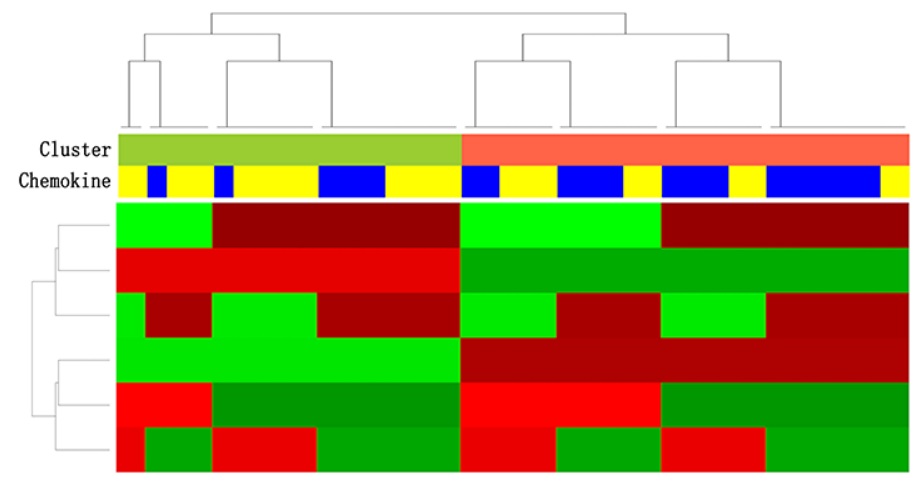

(C)

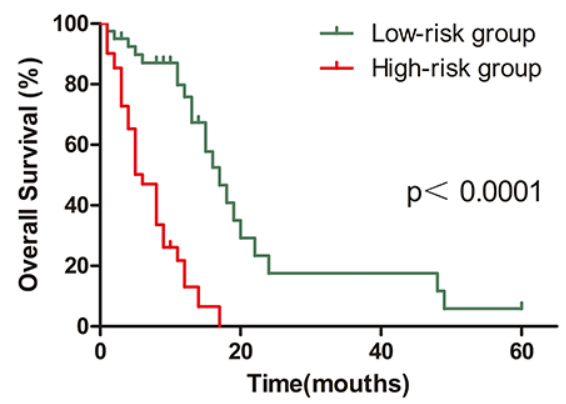

Figure 3: The molecular classification of IDH wild-type astrocytomas. (A) Cluster of Cluster (CoC) Analysis of IDH wildtype astrocytomas. $\mathrm{CoC}$ analysis uses the cluster assignments derived from individual molecular platforms to subdivide tumors, thereby integrating data from analysis of DNA methylation, mRNA, microRNA and lncRNA. For each sample, membership in a particular cluster is indicated by a red tick, and nonmembership is indicated by a green tick. CoC cluster memberships of the tumors are indicated by the color bar: tomato, Cluster CoC $1(\mathrm{n}=12)$; yellowgreen, Cluster CoC $2(\mathrm{n}=11)$. Other color bars indicate various molecular features: blue, high expression of chemokine signaling pathway genes $(n=12)$; yellow, low expression of chemokine signaling pathway genes ( $n=11)$; black, TERT promoter mutation $(\mathrm{n}=16)$; grey, no TERT promoter mutation $(\mathrm{n}=6)$; purple, $1 \mathrm{p} / 19 \mathrm{q}$ codeletion $(\mathrm{n}=5)$; green, no $1 \mathrm{p} / 19 \mathrm{q}$ codeletion $(\mathrm{n}=18)$; brown, GBMs $(\mathrm{n}=15)$; orange, lower-grade astrocytomas $(\mathrm{n}=8)$; pink, high MGMT promoter methylation $(\mathrm{n}=12)$; skyblue, low MGMT promoter methylation $(n=11)$. White indicates missing value. (B) CoC Analyses of IDH wild-type GBM in TCGA dataset. CoC analysis uses the cluster assignments derived from individual molecular platforms to subdivide tumors, thereby integrating data from analysis of DNA methylation, mRNA and microRNA. For each sample, membership in a particular cluster is indicated by a red tick, and nonmembership is indicated by a green tick. CoC cluster memberships of the tumors are indicated by the color bar: tomato, Cluster CoC 1 $(n=47)$; yellowgreen, Cluster CoC $2(n=36)$; blue, high expression of chemokine signaling pathway genes $(n=41)$; yellow, low expression of chemokine signaling pathway genes $(n=42)$. (C) Kaplan-Meier curves of IDH wild-type astrocytoma patients. Patients were classified into high-/low-risk group based on the risk score formula combining signatures involved in chemokine signaling pathway and age. 
in astrocytic tumor tissues compared with non-tumor brain tissues, suggesting that calcium signaling pathway may have critical roles in occurrence and development of astrocytic tumor. Then, the prognostic value of signatures in calcium signaling pathway was verified by risk score method. Astrocytoma patients could be stratified into different tumor risk groups on the basis of risk score combining four signatures and age, and patients in lowrisk group survived significantly longer.

Calcium is the second messenger to regulate different cellular functions including cell proliferation and differentiation, development of neural circuits and axonal guidance. Lots of studies have reported that calcium plays a crucial role in the pathogenesis of many central nervous system diseases, such as Alzheimer's disease [19], Huntington's disease [20]. However, reports about the role of calcium signaling pathway in tumor are uncommon. Polisetty et al. found that calcium binding proteins were observably deregulated in GBM [21], indicating that calcium signaling pathway may be a hallmark of astrocytoma, but its specific function and mechanism in tumorigenesis remain to be further investigated. It might provide potential treatment targets and strategies for astrocytoma patients, and its prognostic value could be validated in prospective and multicenter studies. Additionally, neuroactive ligand-receptor interaction pathway has been reported differentially expressed in diffuse intrinsic pontine glioma (DIPG) [22] and Drosophila Parkinson's disease model [23], it also deserves further research.

Consistant with the previous results [24, 25], IDH mutation was a reliable biomarker of astrocytomas in our research by unsupervised clustering analysis of multiplatform data. We also found that it led to significant change of DNA methylation and RNA expression in complement and coagulation cascades pathway. The complement cascade participates in immune response, and previous studies found that complement pathway could supervise and suppress various cancers. Besides, the expression of complement pathway genes correlated with the outcome of AML patients [26]. The coagulation cascade was activated due to the enhanced vasculature permeability in tumor microenvironment, so it may be a potential target for cancer therapy [27]. But the involvement of complement and coagulation cascades pathway in astrocytoma has not been reported.

In the previous studies of The Cancer Genome Atlas (TCGA), the gene expression of GBM were classified as four subtypes proneural, neural, classical and mesenchymal [28]; and the lower-grade gliomas were subdivided into three subtypes: IDH mutation and $1 \mathrm{p} / 19 \mathrm{q}$ codeletion; IDH mutation and no 1p/19q codeletion; and IDH wild type using the data of multiple platforms [24]. In addition, Eckel-Passow et al defined five molecular groups in 1,087 gliomas using three markers: IDH mutations, $1 \mathrm{p} / 19 \mathrm{q}$ codeletion and TERT promoter mutations [25].
However, the genetic characteristics and molecular classification of biomarkers of astrocytoma remain to be identified, because some alterations are rarely found in astrocytoma, such as $1 \mathrm{p} / 19 \mathrm{q}$ codeletion; and there is still lack of molecular classification research in astrocytoma using multiplatform data.

Furthermore, to determine whether IDH wild-type astrocytoma could be subdivided into groups showing distinct behavior, we conducted a classification by $\mathrm{CoC}$ analysis. We identified two subtypes of IDH wild-type astrocytoma, one was enriched for astrocytomas with TERT promoter mutation and showed high expression of chemokine signaling pathway genes. Moreover, the overall survival of IDH wild-type astrocytoma patients could be predicted by the risk score formula consisting of 3 signatures involved in chemokine signaling pathway and age.

Existing reports showed that chemokine network could promote the invasiveness and angiogenesis of glioma cells [29]; the chemokine receptor CXCR7 contributed to glioma's invasion and angiogenesis, and its expression related to poor outcome in IDH1 mutant patients [30]. Our findings indicate that chemokine signaling pathway plays a fundamental role in highly malignant IDH wildtype astrocytomas, and it can be considered as a potential therapeutic target for astrocytomas.

Taking these together, we discovered that calcium signaling pathway correlated with astrocytoma tumorigenesis and might be a potential biomarker for diagnosis or therapy, then we observed that IDH mutation led to the epigenetic and expression characteristics change in complement and coagulation cascades pathway. Finally, we preliminarily described a molecular classification of IDH wild-type astrocytoma in Chinese population, and the chemokine signaling pathway could be the biomarker.

\section{MATERIALS AND METHODS}

\section{Study population}

This project's protocol was approved by the Ethics Committee of Xiangya School of Medicine, Central South University with registration number of CTXY1300041-3. 33 astrocytic tumor tissues and 9 non-tumor brain tissues were retrospectively obtained from Hunan Province Tumor Hospital and Xiangya Hospital of Central South University (Changsha, Hunan, China) between 2004 and 2011 with informed consent. And all brain tissues were from cerebral cortex. The clinical and pathological data were collected from all patients with histologically confirmed astrocytoma who underwent surgical resection.

\section{Data collection}

Genomic DNA extracted from brain tissues was bisulfite converted using the Zymo EZ DNA Methylation 
Kit (Zymo Research) following the manufacturer's protocols. Genome-wide DNA methylation analysis was performed by Illumina $450 \mathrm{~K}$ Infinium Methylation BeadChip, which quantitatively measures more than 485,000 methylation sites covering 99\% of Ref-Seq genes. The IDAT files were input to $\mathrm{R}$ software by the Bioconductor minfi package to normalize arrays.

Total RNA was isolated from tissue specimens using the TRIzol Reagent (Invitrogen) and purified with NucleoSpin RNA clean-up (MACHEREY- NAGEL) according to the manufacturer's instructions. The quality and integrity of the RNA were verified by gel electrophoresis. The total microRNA was purified using mirVana miRNA Isolation Kit (Ambion) and quantified with NanoDrop ND-1000 spectrophotometer (NanoDrop). The microRNA expression profiling was performed using Human miRNA Microarray, Release 19.0, 8x60K (Agilent Technologies), which contains 2006 human miRNAs. 200 ng of total RNA was labelled and hybridized with miRNA Complete Labeling and Hyb Kit (Agilent Technologies) following the manufacturer's instructions. Slides were scanned by Agilent G2565CA Microarray Scanner and scanned images of microarray were processed with Agilent Feature Extraction Software v10.7 (Agilent Technologies). mRNA and lncRNA expression were measured using LncRNA+mRNA Human Gene Expression Microarray V3.0, 4x180K, which contains 28,844 human mRNAs and 37,581 human lncRNAs. After labeling, hybridization and washing, slides were scanned with the Agilent G2565CA Microarray Scanner (Agilent Technologies). Raw data was extracted through Agilent Feature Extraction Software v10.7 (Agilent Technologies).

\section{Statistical analysis}

The DNA methylation level for each probe is represented as a $\beta$-value ranging from 0 (completely unmethylated) to 1 (completely methylated) $[\beta=$ intensity of the methylated allele (M) / (intensity of the unmethylated allele $(\mathrm{U})+$ intensity of the methylated allele $(\mathrm{M})+100)]$. Probes located on the X or Y chromosomes and probes containing SNPs or "NA" values were excluded prior to the data analyses.

Row data of miRNAs, mRNAs, and lncRNAs was quantile normalized and log 2-scale transformed in GeneSpring GX software (Agilent Technologies). For data quality control, probes located on the $\mathrm{X}$ or $\mathrm{Y}$ chromosomes and probes not reaching a detection rate of $60 \%$ in the upregulated group were excluded. After the filtering procedure, the normalized expression values of mRNA, lncRNA and miRNA probes were median centered, resulted in 17,995 genes, 25,416 lncRNAs and 628 miRNAs for the subsequent analysis respectively.

The two-sided unpaired Student's t-test was performed and the fold changes were calculated between tumor and non-tumor groups. Benjamini and Hochberg method was used to calculate false discovery rate (FDR). Adjusted P-value $<0.05$ and additionally fold change $\geq 2$ were the cut-off value of statistical significance.

\section{qRT-PCR of mRNAs, miRNAs, and IncRNAs}

qRT-PCR was used to validate the expression of mRNAs, miRNAs, and lncRNAs. The total RNA was reverse transcribed to cDNA by PrimeScript RT-PCR Kit (Takara, Dalian, China) according to the manufacturer's protocol. qRT-PCR was performed in Roche LightCycler 480 II Real-Time PCR system (Roche Diagnostics Ltd., Rotkreuz, Switzerland), the relative expression level was calculated using $2^{-\Delta \Delta \mathrm{Ct}}$ method. All primers used are listed in Supplementary Table 1.

\section{Pyrosequencing of IDH mutations}

Pyrosequencing was used to detect the IDH mutation and validate the DNA methylation. Pyrosequencing was performed on PyroMark Q24 system with the Pyro Gold reagents kit according to the manufacturer's protocol, and the result data was analyzed using the PyroMark Q96 software. Detailed information of the pyrosequencing primers is provided in Supplementary Table 2.

\section{Quantitative microsatellite analysis of $1 \mathrm{p} / \mathbf{1 9 q}$ codeletion}

Quantitative microsatellite analysis (QuMA) was performed using 4 microsatellite markers (D1S214, D1S468, D1S2736 and D1S2783) on chromosomes $1 \mathrm{p}$ and 3 microsatellite markers (D19S408, D19S596, and D19S867) on chromosomes $19 \mathrm{q}$ as described previously [31]. And five primer sets for the reference pool were chosen from chromosomes in unaltered gliomas: 3p (D3S1554), 5q (D5S643), 8q (D8S1800), 12q (D12S1699), and 21p (D21S1904) (Supplementary Table 3). The Taqman probe was 5'-FamTGTGTGTGTGTGTGTGTGTGT-Tamra-3'. According to the PCR cycle number $(\mathrm{Ct})$ value, we calculated $\Delta \mathrm{Ct} \quad(\Delta \mathrm{Ct}=\mathrm{Ct} \quad[$ microsatellite]-Ct $\quad[$ reference] $), \Delta \Delta \mathrm{Ct}$ $(\Delta \Delta \mathrm{Ct}=\Delta \mathrm{Ct}$ [tumor]- $\Delta \mathrm{Ct}$ [normal]), and the DNA relative copy number (DNA relative copy number $=2 \times 2^{-\Delta \Delta \mathrm{Ct}}$ ). The $\mathrm{SD}$ and mean value of $\Delta \mathrm{Ct}$ [normal] for each microsatellite marker was used to create a tolerance interval (TI) with a confidence interval of $95 \%$. TI $=2 \pm \mathrm{SD} \times 2.420$, where 2.420 is a two-sided tolerance limit factor for a total number of 70 measurements. Based on the TI, it is defined as a loss or a gain when copy number was less or more than TI, respectively.

\section{MGMT methylation}

A two-probe model (probes cg12434587 and $\operatorname{cg} 12981137)$ was used to determine MGMT methylation. 
M-value of each probe could be calculated by the equation: The equation for this model is given below:

$$
\mathrm{M}=\log 2\left(\frac{\text { Beta }}{1-\text { Beta }}\right)
$$

$\operatorname{logit}(\mathrm{y})=4.3215+0.5271^{*} \mathrm{M} \quad(\operatorname{cg} 12434587)+$

$0.9265^{*} \mathrm{M}(\operatorname{cg} 12981137)$

The methylation probability y was computed using the inverse logit function, and the cut-off value was 0.358 .

\section{Author contributions}

Conceived and designed the experiments: JYY, HHZ, ZQL. Performed the experiments: CXM, YZ, ZBW, XYM. Analyzed the data: CXM, JYY, XL. Contributed reagents/materials/analysis tools: ZQY, ZWH, XML, XJL, WZ. Wrote the paper: CXM, JYY, RTC, ZQL. Reviewed the manuscript: all authors.

\section{CONFLICTS OF INTEREST}

The authors declare no conflicts of interest.

\section{FUNDING}

This work was supported by the National Key Research and Development Programs (2016YFC1306900, 2016YFC0905002), National Natural Science Foundation of China $(81373490,81573463,81573508)$, and The Strategy-Oriented Special Project of Central South University in China (ZLXD2017003).

\section{REFERENCES}

1. Ostrom QT, Gittleman H, Fulop J, Liu M, Blanda R, Kromer C, Wolinsky Y, Kruchko C, Barnholtz-Sloan JS. CBTRUS Statistical report: primary brain and central nervous system tumors diagnosed in the united states in 2008-2012. Neuro Oncol. 2015; 17:iv1-iv62.

2. Louis DN, Ohgaki H, Wiestler OD, Cavenee WK, Burger PC, Jouvet A, Scheithauer BW, Kleihues P. The 2007 WHO classification of tumours of the central nervous system. Acta Neuropathol. 2007; 114:97-109.

3. Wen PY, Kesari S. Malignant gliomas in adults. N Engl J Med. 2008; 359:492-507.

4. Coons SW, Johnson PC, Scheithauer BW, Yates AJ, Pearl $\mathrm{K}$. Improving diagnostic accuracy and interobserver concordance in the classification and grading of primary gliomas. Cancer. 1997; 79:1381-1393.

5. Yan Y, Xu Z, Li Z, Sun L, Gong Z. An insight into the increasing role of LncRNAs in the pathogenesis of gliomas. Front Mol Neurosci. 2017; 10:53.

6. Li Z, Zhou Q, Li Y, Yan S, Fu J, Huang X, Shen L. Mean cerebral blood volume is an effective diagnostic index of recurrent and radiation injury in glioma patients: a metaanalysis of diagnostic test. Oncotarget. 2017; 8:1564215650. https://doi.org/10.18632/oncotarget.14922.

7. Parsons DW, Jones S, Zhang X, Lin JC, Leary RJ, Angenendt P, Mankoo P, Carter H, Siu IM, Gallia GL, Olivi A, McLendon R, Rasheed BA, et al. An integrated genomic analysis of human glioblastoma multiforme. Science. 2008; 321:1807-1812.

8. Balss J, Meyer J, Mueller W, Korshunov A, Hartmann C, von Deimling A. Analysis of the IDH1 codon 132 mutation in brain tumors. Acta Neuropathol. 2008; 116:597-602.

9. Yan H, Parsons DW, Jin G, McLendon R, Rasheed BA, Yuan W, Kos I, Batinic-Haberle I, Jones S, Riggins GJ, Friedman H, Friedman A, Reardon D, et al. IDH1 and IDH2 mutations in gliomas. N Engl J Med. 2009; 360:765-773.

10. Bello MJ, Vaquero J, de Campos JM, Kusak ME, Sarasa JL, Saez-Castresana J, Pestana A, Rey A. Molecular analysis of chromosome 1 abnormalities in human gliomas reveals frequent loss of $1 \mathrm{p}$ in oligodendroglial tumors. Int J Cancer. 1994; 57:172-175.

11. Brennan CW, Verhaak RG, McKenna A, Campos B, Noushmehr H, Salama SR, Zheng S, Chakravarty D, Sanborn JZ, Berman SH, Beroukhim R, Bernard B, Wu CJ, et al. The somatic genomic landscape of glioblastoma. Cell. 2013; 155:462-477.

12. Hegi ME, Diserens AC, Gorlia T, Hamou MF, de Tribolet N, Weller M, Kros JM, Hainfellner JA, Mason W, Mariani L, Bromberg JE, Hau P, Mirimanoff RO, et al. MGMT gene silencing and benefit from temozolomide in glioblastoma. N Engl J Med. 2005; 352:997-1003.

13. Berghoff AS, Hainfellner JA, Marosi C, Preusser M. Assessing MGMT methylation status and its current impact on treatment in glioblastoma. CNS Oncol. 2015; 4:47-52.

14. Ferreira MA, O'Donovan MC, Meng YA, Jones IR, Ruderfer DM, Jones L, Fan J, Kirov G, Perlis RH, Green EK, Smoller JW, Grozeva D, Stone J, et al. Collaborative genome-wide association analysis supports a role for ANK3 and CACNA1C in bipolar disorder. Nat Genet. 2008; 40:1056-1058.

15. Iqbal Z, Vandeweyer G, van der Voet M, Waryah AM, Zahoor MY, Besseling JA, Roca LT, Vulto-van Silfhout AT, Nijhof B, Kramer JM, Van der Aa N, Ansar M, Peeters H, et al. Homozygous and heterozygous disruptions of ANK3: at the crossroads of neurodevelopmental and psychiatric disorders. Hum Mol Genet. 2013; 22:1960-1970.

16. Epping MT, Meijer LA, Krijgsman O, Bos JL, Pandolfi PP, Bernards R. TSPYL5 suppresses p53 levels and function by physical interaction with USP7. Nat Cell Biol. 2011; 13:102-108.

17. Qiu X, Hu B, Huang Y, Deng Y, Wang X, Zheng F. Hypermethylation of ACP1, BMP4, and TSPYL5 in hepatocellular carcinoma and their potential clinical significance. Dig Dis Sci. 2016; 61:149-157.

18. Wollmer MA, Kapaki E, Hersberger M, Muntwyler J, Brunner F, Tsolaki M, Akatsu H, Kosaka K, Michikawa M, 
Molyva D, Paraskevas GP, Lutjohann D, von Eckardstein A, et al. Ethnicity-dependent genetic association of ABCA2 with sporadic Alzheimer's disease. Am J Med Genet B Neuropsychiatr Genet. 2006; 141B:534-536.

19. Berridge MJ. Calcium signalling and Alzheimer's disease. Neurochem Res. 2011; 36:1149-1156.

20. Bezprozvanny I, Hayden R. Deranged neuronal calcium signaling and Huntington disease. Biochem Biophys Res Commun. 2004; 322:1310-1317.

21. Polisetty RV, Gautam P, Sharma R, Harsha HC, Nair SC, Gupta MK, Uppin MS, Challa S, Puligopu AK, Ankathi P, Purohit AK, Chandak GR, Pandey A, et al. LC-MS/MS analysis of differentially expressed glioblastoma membrane proteome reveals altered calcium signaling and other protein groups of regulatory functions. Mol Cell Proteomics. 2012; 11:M111 013565.

22. Deng L, Xiong $\mathrm{P}$, Luo $\mathrm{Y}, \mathrm{Bu} \mathrm{X}$, Qian S, Zhong W. Bioinformatics analysis of the molecular mechanism of diffuse intrinsic pontine glioma. Oncol Lett. 2016; 12:2524-2530.

23. Kong Y, Liang X, Liu L, Zhang D, Wan C, Gan Z, Yuan L. High throughput sequencing identifies microRNAs mediating alpha-synuclein toxicity by targeting neuroactive-ligand receptor interaction pathway in early stage of drosophila Parkinson's disease model. PLoS One. 2015; 10:e137432.

24. Brat DJ, Verhaak RG, Aldape KD, Yung VK, Salama SR, Cooper LA, Rheinbay E, Miller CR, Vitucci M, Morozova O, Robertson AG, Noushmehr H, Laird PW, et al, and Cancer Genome Atlas Research Network. Comprehensive, integrative genomic analysis of diffuse lower-grade gliomas. N Engl J Med. 2015; 372:2481-2498.

25. Eckel-Passow JE, Lachance DH, Molinaro AM, Walsh KM, Decker PA, Sicotte H, Pekmezci M, Rice T, Kosel ML,
Smirnov IV, Sarkar G, Caron AA, Kollmeyer TM, et al. Glioma groups based on 1p/19q, IDH, and TERT promoter mutations in tumors. N Engl J Med. 2015; 372:2499-2508.

26. Laverdiere I, Boileau M, Herold T, Rak J, Berdel WE, Wormann B, Hiddemann W, Spiekermann K, Bohlander SK, Eppert K. Complement cascade gene expression defines novel prognostic subgroups of acute myeloid leukemia. Exp Hematol. 2016; 44:1039-1043.e1010.

27. Liu Y, Jiang P, Capkova K, Xue D, Ye L, Sinha SC, Mackman N, Janda KD, Liu C. Tissue factor-activated coagulation cascade in the tumor microenvironment is critical for tumor progression and an effective target for therapy. Cancer Res. 2011; 71:6492-6502.

28. Verhaak RG, Hoadley KA, Purdom E, Wang V, Qi Y, Wilkerson MD, Miller CR, Ding L, Golub T, Mesirov JP, Alexe G, Lawrence M, O'Kelly M, et al. Integrated genomic analysis identifies clinically relevant subtypes of glioblastoma characterized by abnormalities in PDGFRA, IDH1, EGFR, and NF1. Cancer Cell. 2010; 17:98-110.

29. Domanska UM, Kruizinga RC, den Dunnen WF, TimmerBosscha H, de Vries EG, Walenkamp M. The chemokine network, a newly discovered target in high grade gliomas. Crit Rev Oncol Hematol. 2011; 79:154-163.

30. Birner P, Tchorbanov A, Natchev S, Tuettenberg J, Guentchev M. The chemokine receptor CXCR7 influences prognosis in human glioma in an IDH1-dependent manner. J Clin Pathol. 2015; 68:830-834.

31. Nigro JM, Takahashi MA, Ginzinger DG, Law M, Passe $\mathrm{S}$, Jenkins RB, Aldape K. Detection of $1 \mathrm{p}$ and $19 \mathrm{q}$ loss in oligodendroglioma by quantitative microsatellite analysis, a real-time quantitative polymerase chain reaction assay. Am J Pathol. 2001; 158:1253-1262. 